\title{
A OPINIÃO DE ENFERMEIROS RECÉM-ADMITIDOS SOBRE O PROGRAMA DE TREINAMENTO PARA A SUA INTEGRAÇÃO EM UM HOSPITAL DE ENSINO
}

\author{
Teresa Cristina Manrique Coan* \\ Vera Lúcia Mira Gonçalves** \\ Maria Madalena Januário Leite*** \\ Valéria Castilho****
}

COAN, T.C.M. et al. A opinião de enfermeiros recém-admitidos sobre o programa de treinamento para a sua integração em um hospital de ensino. Rev.Esc.Enf.USP, v.30, n.2, p.187-203, ago. 1996.

As autoras propuseram-se a conhecer a opinião dos enfermeiros recém-admitidos em um hospital de ensino, sobre o treinamento realizado para a sua integração, à fim de obter subsidios para a avaliação e reformulação do mesmo.

UNITERMOS: Treinamento de enfermeiros. Programa de integração de enfermeiros.

\section{INTRODUÇÃO}

A adaptação de novos funcionários em locais de trabalho cada vez mais complexos tem sido um dos desafios enfrentados pelas organizações.

Para facilitar essa adaptação as organizações que contratam grande números de funcionários têm adotado programas formais de orientação dos recém-admitidos.

\footnotetext{
* Enfermeira do Serviço de Apoio Educacional do Departamento de Enfermagem do Hospital Universitário da USP.

* Enfermeira - Diretora do Serviço de Apoio Educacional do Departamento de Enfermagem do Hospital Universitário da USP, mestranda do Programa de PósGraduação da EEUSP na área de Administração em Serviço de Enfermagem.

*** Enfermeira - Professor Doutor da Disciplina de Administração Aplicada à Enfermagem do Departamento de Orientação Profissional da Escola de Enfermagem da USP. Representante da EEUSP nos processos seletivos de enfermeiros do Hospital Universitário da USP.

**** Enfermeira - Professora Assistente da Disciplina de Administração Aplicada à Enfermagem do Departamento de Orientação Profissional da Escola de Enfermagem da USP. Coordenadora de Ensino e Pesquisa da área de Educação Continuada do Departamento de Enfermagem do Hospital Universitário da USP.
} 
Esses programas têm recebido diferentes denominações: programa de orientação ( WERTHER; DAVIS ${ }^{9}$ SILVA; PEREIRA; BENKO ${ }^{8}$ ), programa de orientação inicial (LEITE, PEREIRA ${ }^{6}$ ), treinamento prévio (SANTOS ${ }^{7}$ ), treinamento de indução (CHIAVENATO ${ }^{3}$ ), programa de ambientação (AQUINO ${ }^{1}$ ) e programa de integração (CHIAVENATO ${ }^{3}$, BERGSTEIN ${ }^{2}$ ).

Embora recebam nomes diferentes eles se referem a um conjunto de práticas que, ao nível de senso comum significam "ajustar", "facilitar", "informar" e "sensibilizar" o recém-admitido à empresa (BERGSTEIN²).

WHERTHER; DAVIS ${ }^{9}$ justificam a adoção de programas de orientação afirmando que, "embora os recém-admitidos estejam qualificados para o trabalho, quando passam por um processo de seleção há necessidade de compreenderem os procedimentos e as políticas da organização, conhecerem as demais pessoas com quem trabalham e serem treinadas para executar as suas funções”.

Esses programas têm a finalidade de informar o novo empregado sobre vários aspectos da vida funcional e social da instituição. Falhas nessa etapa podem comprometer o exercício de uma atividade criando dificuldades no desempenho profissional (SANTOS ${ }^{7}$ ). Geralmente esses programas possuem duas fases: a integração do funcionário novo na organização e a integração do funcionário novo no setor de trabalho.

As aulas da primeira fase são geralmente ministradas pelos profissionais de recursos humanos, que transmitem informações sobre a história e a filosofia da empresa, sua estrutura organizacional, normas e regulamentos, planta física, direitos e deveres do pessoal, os benefícios, a descrição e os deveres do cargo.

Na segunda fase, o chefe do setor onde o funcionário novo será alocado assume a responsabilidade de integrá-lo, orientando-o sobre a instituição, apresentando-o na equipe e auxiliando-o na aquisição de conhecimentos específicos tanto sobre sua função, como sobre a estrutura e o funcionamento do setor.

Geralmente esse treinamento inicial ocorre no período de um a três meses.

WERTHER; DAVIS ${ }^{9}$ afirmam que, embora as pesquisas sobre programas de orientação sejam limitadas, alguns benefícios são geralmente reportados, tais como: a diminuição da sensação de isolamento, diminuição da apreensão e ansiedade que tornam os indivíduos menos aptos a aprender; por outro lado dão mais segurança aos indivíduos e com isso estes necessitam de menos atenção de colegas e supervisores; há também diminuição de críticas e repreensões dos superiores, o que os tornam mais depressa parte da organização e menos inclinados a se demitirem.

Para CHIAVENATO ${ }^{3}$, os programas de integração reduzem o período de adaptação ao trabalho, fornecem ao elemento supervisor oportunidade de 
orientar o novo funcionário corretamente, antes que outro o faça da forma errada; diminuem a necessidade de ações corretivas e penalidades devido ao desconhecimento dos regulamentos da empresa; fornecem subsídios para o empregado vencer o medo do desconhecido que dificulta o alcance da produção ideal e diminuem o número de demissões.

Fica claro que o objetivo dos programas de integração de novos funcionários é a fixação dos mesmos em seus locais de trabalho, uma vez que altos índices de "turnover" representam custos elevados para a organização.

Segundo $\mathrm{AQUINO}^{1}$, a política de integração das empresas tem por objetivo propiciar ao trabalhador condições satisfatórias de trabalho, a fim de torná-lo uma pessoa satisfeita e, consequentemente, produtiva.

Embora um programa de treinamento possa tornar-se eficaz, se for bem organizado, há necessidade de ser verificado se ele está atingindo seu objetivo e se as estratégias empregadas estão sendo eficazes.

Precisa-se verificar se os benefícios esperados com a adoção de programas de treinamento de integração estão ocorrendo de fato, isto é, se estão facilitando a adaptação dos novos funcionários a seus locais de trabalho.

Com esse objetivo, nós enfermeiras da Educação Continuada do Hospital Universitário, em conjunto com docentes da Escola de Enfermagem da Universidade de São Paulo, nos propusemos a avaliar o programa de treinamento inicial para enfermeiros que ingressam na Instituição, por ser essa a categoria que tem o maior índice de rotatividade no Hospital.

Tal programa tem sido denominado de "treinamento admissional" e foi elaborado há 12 anos, tendo sofrido, ao longo desse período, algumas modificações.

Atualmente é desenvolvido em duas fases.

A primeira é ministrada na Educação Continuada do Serviço de Apoio Educacional do Departamento de Enfermagem do HU, em nove dias úteis, 6 horas diárias. Em relação ao conteúdo dessa fase existe um modelo de treinamento padrão que é adaptado às necessidades do grupo que está sendo admitido, à disponibilidade dos ministrantes das aulas e à distribuição dos temas no período em que será realizado. (ANEXO 1).

$\mathrm{Na} 2 \mathrm{a}$ fase, realizada na unidade onde o enfermeiro é lotado, o treinamento específico tem a duração de 30 dias e e feito no período da manhã. Para isso, é seguido o cronograma de orientação específica, que é adaptado para as diferentes unidades do Hospital (ANEXO 2), acompanhado pela chefe de seção ou por uma colega mais experiente.

Os objetivos do programa de treinamento admissional foram os seguintes: contribuir para maior segurança do paciente, maior tranquilidade do funcionário em relação ao ambiente de trabalho e para que os objetivos da instituição sejam atingidos da maneira mais econômica possível; reduzir os riscos de acidente de trabalho e zelar pelo patrimônio da instituição. 
Iniciada a avaliação do processo de integração dos enfermeiros no Hospital, foi verificado que tal processo era bastante complexo. Houve necessidade de cruzamento de várias informações referentes à opinião dos recém-admitidos, das enfermeiras chefes e da equipe de trabalho sobre o programa desenvolvido, bem como das informações obtidas nas entrevistas de desligamento de enfermeiros recém-contratados e aos resultados das avaliações de seu desempenho nesse período.

Optamos por iniciar a análise desse processo obtendo informações através da sondagem de opinião dos enfermeiros novos que haviam passado recentemente pelo treinamento inicial. Para FERNANDES ${ }^{5}$," o levantamento e a análise das opiniões permitem captar dados relativos às motivações e as atitudes dos trabalhadores, bem como subsidiar decisões quanto às políticas e estratégias organizacionais".

Este estudo sobre a opinião dos enfermeiros recém-admitidos, a respeito do treinamento realizado para a sua integração a um hospital de ensino, é apenas o início de uma longa jornada, que possibilitará uma avaliação global do processo de integração do enfermeiro ao Hospital.

\section{OBJETIVOS}

- Conhecer a opinião dos enfermeiros recém-admitidos sobre o programa de treinamento para sua integração em um hospital de ensino.

- Obter subsídios para a avaliação e reformulação do programa de treinamento para a integração de enfermeiros recém-admitidos, ministrado pela Educação Continuada do Departamento de Enfermagem de um hospital de ensino.

\section{METODOLOGIA}

\subsection{LOCAL DO ESTUDO}

O presente estudo foi realizado no Hospital Universitário da Universidade de São Paulo, na cidade de São Paulo.

\subsection{POPULAÇÃO}

A população inicial foi constituída de 12 enfermeiros que prestaram concurso e foram admitidos no hospital em $1^{\circ}$ de Abril de 1993 e que passaram 
pelo programa de treinamento admissional havia 2 meses. Porém, apenas 8 enfermeiros participaram deste estudo, pois os demais não entregaram o questionário para a coleta de dados.

Esses 8 enfermeiros estão lotados em diferentes unidades do hospital a saber: 1 no Berçário, 3 na Emergência, 2 no Centro Obstétrico e 2 na Clínica Médica .

Os dados para a caracterização da população do estudo foram obtidos no currículo de cada enfermeiro, o que é exigido para recrutamento e seleção.

\subsection{COLETA DE DADOS}

Os dados foram obtidos por meio de um questionário (ANEXO 3), entregue à população alvo pelas enfermeiras responsáveis pela Educação Continuada, após a explicação dos objetivos do mesmo e a anuência das enfermeiras.

O questionário contém oito perguntas relacionadas à fase do treinamento desenvolvido na Educação Continuada e seis referentes a segunda fase do treinamento realizado nas unidades onde as enfermeiras admitidas haviam sido lotadas.

\subsection{ANÁLISE DOS DADOS}

Os dados obtidos por meio dos questionários foram analisados conforme sua frequência absoluta e relativa. As justificativas e sugestões dadas pelos enfermeiros para cada questão foram agrupadas de acordo com suas semelhanças.

\section{DISCUSSÃo DOS RESULTADOS}

\subsection{A CARACTERIZAÇÃo da POPULAÇÃo}

A idade da população estudada variou de 23 a 43 anos, sendo predominante a faixa entre 23 e 29 anos $(62,5 \%)$; quanto ao sexo, houve predomínio do feminino $(87,5 \%)$.

O tempo de formado variou de 4 meses a 8 anos e 4 meses, predominando enfermeiros graduados entre 4 meses e 2 anos e 4 meses de formados (75\%).

Foi verificado que $37,5 \%$ eram recém-formados e que $50 \%$ dos enfermeiros não tinham nenhuma experiência profissional anterior. 


\subsection{A OPINIÃO DOS ENFERMEIROS RECÉM-ADMITIDOS EM RELAÇÃO À FASE DO TREINAMENTO REALIZADO NA EDUCAÇÃO CONTINUADA}

Em relação ao conteú do do programa 100\% responderam que os temas abordados contribuiram para a sua adaptação inicial nas unidades onde foram lotados.

Tabela 1 - Distribuição da opinião dos enfermeiros recém-admitidos sobre a contribuição do conteúdo do Programa de Treinamento para a sua integração. São Paulo, 1993.

\begin{tabular}{lcc}
\hline \multicolumn{1}{c}{ Opinião } & $\mathrm{N}^{\circ}$ & $\%$ \\
\hline $\begin{array}{l}\text { Possibilitou o conhecimento da filosofia, } \\
\text { normas e rotinas da Instituição e do } \\
\text { Departamento de Enfermagem. }\end{array}$ & 07 & 87,50 \\
$\begin{array}{l}\text { Propiciou a aquisição de segurança pela } \\
\text { compreensão do papel esperado do } \\
\text { enfermeiro }\end{array}$ & 01 & 12,50 \\
\hline TOTAL & 08 & 100,00 \\
\hline \hline
\end{tabular}

Foi verificada a importância que os enfermeiros atribuem ao conhecimento da filosofia e das normas da Instituição para sua adaptação ao trabalho (87,5\%). Esse conhecimento oferece segurança aos elementos que ingressam numa organização, que ficam conhecendo a finalidade e os objetivos organizacionais, bem como o que compete aos funcionários em relação a direitos e deveres.

Apesar dos enfermeiros considerarem que o treinamento inicial desenvolvido na Educação Continuada, antes de serem lotados em suas unidades, os havia auxiliado na sua adaptação, ao serem questionados se esse treinamento havia sido o esperado, segundo a tabela 2, mostrada a seguir, a grande maioria considerou não ter correspondido às suas expectativas. 
Tabela 2 - Distribuição da opinião dos enfermeiros recém-admitidos quanto ao atendimento de suas expectativas pelo treinamento desenvolvido pela Educação Continuada. São Paulo, 1993.

\begin{tabular}{lcc}
\hline \multicolumn{1}{c}{ Opinião } & $\mathbf{n}^{\mathbf{0}}$ & $\%$ \\
\hline FAVORÁVEL & 2 & 25,00 \\
$\begin{array}{l}\text { Forneceu conhecimentos prévios } \\
\text { importantes para a adaptação } \\
\text { na Unidade }\end{array}$ & 1 & \\
$\begin{array}{l}\text { Favoreceu a discussão e troca } \\
\text { de experiência entre o grupo } \\
\text { e os enfermeiros da Educação }\end{array}$ & & 12,50 \\
Continuada & 3 & 37,50 \\
\hline SUBTOTAL & & \\
\hline
\end{tabular}

\section{DESFAVORÁVEL}

Tempo reduzido

Não abordou todos os temas

necessários

Deveria ter sido desenvolvido

paralelamente ao treinamento da unidade

Os dados mostram que $62,5 \%$ dos enfermeiros não ficaram satisfeitos quanto ao atendimento de suas expectativas pelos motivos acima expostos. $\mathrm{E}$ de supor que a necessidade sentida do programa ser ministrado concomitantemente ao da unidade é devida à necessidade dos enfermeiros de resgatarem as informações téricas obtidas na Educação Continuada confrontando-as com aquilo que vivenciaram na unidade. É sabido que, muitas vezes normas, rotinas e procedimentos sofrem modificações no dia a dia. Tal fato gera ansiedade e insegurança nos elementos novos que não sabem se seguem as orientações dadas na Educação Continuada ou a que recebem na Unidade. 
A próxima tabela mostra os temas que foram apontados como aqueles que poderiam ser acrescentados ao programa de treinamento .

Tabela 3 - Distribuição dos temas a serem incluídos no treinamento desenvolvido pela Educação Continuada segundo a opinião dos enfermeiros recém-admitidos. São Paulo, 1993

\begin{tabular}{llc}
\hline \multicolumn{1}{c}{ Temas } & $\mathrm{N}^{\mathrm{O}}$ & $\%$ \\
\hline Procedimentos específicos da Unidade & 01 & 12,50 \\
$\begin{array}{l}\text { Normas e rotinas específicas da Uni- } \\
\text { dade }\end{array}$ & 01 & 12,50 \\
$\begin{array}{l}\text { Leitura e manuseio dos manuais de } \\
\text { procedimentos }\end{array}$ & 01 & 12,50 \\
$\begin{array}{l}\text { Relações humanas na família, no traba- } \\
\text { lho e na sociedade }\end{array}$ & 01 & 12,50 \\
\hline TOTAL & 08 & 100,00 \\
\hline \hline
\end{tabular}

Embora não seja objetivo da primeira fase do treinamento, fornecer informações sobre normas e rotinas especificas das unidades, bem como leitura de manuais de procedimentos, pois esses assuntos deveriam ser vistos nas Unidades, essa foi uma necessidade sentida por $25 \%$ dos enfermeiros.

As autoras do presente trabalho compreendem que essa necessidade deve ser atendida pois o conhecimento das normas e rotinas do trabalho oferecem segurança aos elementos que ingressam numa organização, principalmente aos profissionais recém-formados, pois os auxiliam nas tomadas de decisões.

Quanto ao tempo programado para a realização do programa na Educação Continuada, os enfermeiros apresentaram a sua opinião como podemos verificar na tabela 4 .

Tabela 4 - Distribuição da relação dos temas que, segundo os enfermeiros recém-admitidos, devem ter a carga horária aumentada no treinamento. São Paulo, 1993.

Temas

$\mathrm{N}^{\mathrm{O}} \%$

Sistema HMS (informatização)

$05 \quad 62,50$

Sistema de Assistência de Enfermagem 02

Supervisão e Liderança

01

25,00

12,50

TOTAL

08

100,00 
A maioria $(62,5 \%)$, gostaria que o sistema de informatização (HMS) fosse desenvolvido por um tempo maior. Na verdade, esse tema é desenvolvido em 4 horas, porém, especificamente neste treinamento, por impossibilidade da pessoa responsável, ele foi de sòmente uma hora, o que demonstra a necessidade de se dar esse assunto em um período maior.

Quanto ao Sistema de Assistência de Enfermagem (SAE) 25\% consideraram o tempo insuficiente, sugerindo que fossem aumentadas as horas para a aula de exame físico e maior tempo para os exercícios práticos do SAE, ainda que a grande maioria relatou que o conteudo foi satisfatório.

Também verificamos a opinião dos enfermeiros quanto as estratégias sendo que a sua mioria considerou-as adequadas aos temas, pois facilitaram a troca de experiências entre os profissionais envolvidos no treinamento.

A tabela 5 mostra que a maioria dos enfermeiros consideraram boa a interação com os enfermeiros da Educação Continuada.

Tabela 5 - Distribuição da opinião dos enfermeiros recém-admitidos sobre a interação do grupo de treinandos com as enfermeiras da Educação Continuada. São Paulo, 1993.

\begin{tabular}{lll}
\hline Opinião & $\mathrm{N}^{\mathrm{O}}$ & $\%$ \\
\hline
\end{tabular}

FAVORAVEL

Houve apoio e abertura aos questiona-

05

62,50

mentos dos novos elementos

Possibilitou o alcance dos objetivos

01

12,50

do treinamento

Serviu como elo para a integração

01

12,50

\begin{tabular}{lll}
\hline SUBTOTAL & 07 & 87,50
\end{tabular}

DESFAVORÁVEL

Houve dificuldade de atuação e adaptação

01

12,50

devido às expectativas e ansiedade do

grupo de treinandos

TOTAL

08

100,00

Segundo os dados obtidos, foi ressaltada a importância da atuação dos enfermeiros da Educação Continuada no processo de integração de elementos novos na instituição. O primeiro contato com a organização é mediada por tais elementos, que sofrem o primeiro impacto da ansiedade do grupo, por esse motivo os enfermeiros de educação Continuada necessitam de preparo para lidar com esse aspecto. 
Confirmando essa opinião 2 treinandos sugeriram que o enfermeiro da Educação Continuada acompanhe os novos elementos nas unidades, pelo menos nos primeiros dias, e que haja uma avaliação semanal com os mesmos durante todo o período de treinamento.

\subsection{OPINIÃO DOS ENFERMEIROS RECÉM-ADMITIDOS SOBRE A FASE DO TREINAMENTO DESENVOLVIDO NA UNIDADE}

Nos dados descritos na tabela 6, a maioria dos enfermeiros consideraram que o treinamento facilitou o conhecimento da dinâmica de trabalho na Unidade.

Tabela 6 - Distribuição da opinião dos enfermeiros recém-admitidos quanto ao treinamento oferecido na unidade. São Paulo, 1993.

\begin{tabular}{lll}
\hline \hline Opinião & No & $\%$ \\
\hline FAVORAVEL & & \\
$\begin{array}{l}\text { Facilitou o conhecimento da dinâ- } \\
\text { mica da unidade }\end{array}$ & 05 & 62,50 \\
\hline SUBTOTAL & 05 & 62,50 \\
\hline
\end{tabular}

\section{DESFAVORÁVEL}

As normas e as rotinas da Unidade não estão escritas

01

01

Faltou tempo para completar o treinamento

"Só se aprende com os apuros"
01

12,50

\begin{tabular}{lll}
\hline SUBTOTAL & 03 & 37,50 \\
\hline TOTAL & 08 & 100,00 \\
\hline \hline
\end{tabular}

Constatamos pelo resultado desta tabela que a maioria dos enfermeiros perceberam de forma favorável o treinamento $(62,50 \%)$ e os demais $37,50 \%$ apontaram alguns aspectos a serem melhorados referindo-se a questão das normas e rotinas e ao tempo ser insuficiente para o treinamento. Cabe ressaltar que embora exista o manual de normas e rotinas nas unidades, este se encontra em fase de atualização o que tem dificultado a sua utilização durante o treinamento. 
Tabela 7 - Distribuição da opinião dos enfermeiros recém-admitidos sobre o período de treinamento na Unidade. São Paulo, 1993

\begin{tabular}{lcc}
\hline \multicolumn{1}{c}{ Opinião } & $\mathrm{N}^{\mathrm{O}}$ & $\%$ \\
\hline \multicolumn{1}{c}{ FAVORÁVEL } & & 12,50 \\
$\begin{array}{l}\text { Foi suficiente para o conhecimento } \\
\text { da dinâmica, mas algumas situações } \\
\text { não ocorreram neste período }\end{array}$ & 01 & 12,50 \\
$\begin{array}{l}\text { Foi suficiente embora seja necessá } \\
\text { rio continuar o aprendizado }\end{array}$ & 01 & 25,00 \\
\hline $\begin{array}{l}\text { SUBTOTAL } \\
\text { DESFAVORÁVEL }\end{array}$ & 02 & 75,00 \\
$\begin{array}{l}\text { O tempo é insuficiente para a com- } \\
\text { plexidade da unidade }\end{array}$ & 06 & 100,00 \\
\hline TOTAL & 08 & 1 \\
\hline \hline
\end{tabular}

Os dados desta tabela mostram que $75 \%$ dos enfermeiros consideraram o tempo insuficiente para o treinamento na unidade. Realmente, o período de um mês é um prazo curto para o conhecimento da dinâmica de uma unidade devido à complexidade desta. O que se espera é que nesse período o enfermeiro tenha adquirido o mínimo de informações necessárias para sua adaptação e o seguimento seja oferecido pelo grupo da unidade.

A tabela abaixo mostra como os enfermeiros consideraram a utilização de um cronograma de orientação específica (ANEXO 2) .

Tabela 8 - Distribuição da opinião dos enfermeiros recém-admitidos sobre a utilização do cronograma de orientação específica para o conhecimento das Unidades. São Paulo, 1993.

\begin{tabular}{|c|c|c|}
\hline Opinião & $\mathrm{N}^{\mathrm{O}}$ & $\%$ \\
\hline \multicolumn{3}{|l|}{ FAVORÁVEL } \\
\hline $\begin{array}{l}\text { Serve como guia impedindo que algo } \\
\text { seja esquecido }\end{array}$ & 05 & 62,50 \\
\hline SUBTOTAL & 05 & 62,50 \\
\hline \multicolumn{3}{|l|}{ DESFAVORÁVEL } \\
\hline $\begin{array}{l}\text { O conhecimento foi adquirido no } \\
\text { dia a dia }\end{array}$ & 01 & 12,50 \\
\hline $\begin{array}{l}\text { Não há necessidade de reavaliar os } \\
\text { procedimentos das pessoas que já } \\
\text { passaram no concurso de seleção }\end{array}$ & 01 & 12,50 \\
\hline $\begin{array}{l}\text { O guia } 6 \text { específico sobre a Unidade; } \\
\text { nada tem a ver com o treinamen } \\
\text { to dado pela Educação Continuada }\end{array}$ & 01 & 12,50 \\
\hline SUBTOTAL & 03 & 37,50 \\
\hline TOTAL & 08 & 100,00 \\
\hline
\end{tabular}


Os dados mostram que a maioria dos enfermeiros, isto é, $62,50 \%$ consideraram importante a utilização de um cronograma de orientação especifica.

Em relação a enfermeira que referiu ser o cronograma de orientação muito específico percebe-se que ela sentiu uma ruptura na continuidade do treinamento, como se aquilo que foi desenvolvido na Educação Continuada não fosse uma preparação para a unidade. Esta enfermeira sugeriu que alguém da Educação Continuada acompanhasse os treinandos nas unidades.

A utilização de um cronograma com todas as rotinas e procedimentos a serem executados pelos treinandos foi considerado por uma enfermeira $(12,5 \%)$ como uma "avaliação" desnecessária, uma vez que já fora aprovada no concurso. Sugere que o novo enfermeiro converse sobre o que sabe e não sabe com a sua chefia, antes de seguir o cronograma.

Tabela 9 - Distribuição da opinião dos enfermeiros recém-admitidos quanto ao acompanhamento de um enfermeiro na Unidade. São Paulo, 1993.

\begin{tabular}{lll}
\hline Opinião & $\mathrm{N}^{\mathrm{O}}$ & $\%$
\end{tabular}

FAVORÁVEL

O enfermeiro da Unidade torna-se um

07

87,50

ponto de referência para esclareci-

mento de dúvidas e para avaliação

da nossa atuação

\begin{tabular}{lll}
\hline SUBTOTAL & 07 & 87,50
\end{tabular}

DESFAVORÁVELl

Os enfermeiros das unidades estão *

01

12,50

tão sobrecarregados, pelo serviço que

temos a sensação que os estamos atrapa-

lhando

\begin{tabular}{lcc}
\hline SUBTOTAL & 01 & 12,50 \\
\hline \hline TOTAL & 08 & 100,00 \\
\hline \hline
\end{tabular}

A tutela de um colega mais experiente é vista como favorável pela maioria $(87,5 \%)$ dos novos enfermeiros, conforme mostra a tabela 9.

BERGSTEIN ${ }^{2}$ acredita que a supervisão do novo funcionário por um colega de trabalho facilita ao novato seus contatos humanos no grupo e a execução de tarefas específicas. Porém, é necessário ter em mente o número de novos funcionários a serem integrados e a disponibilidade de tempo, pessoal, instalação e de outros recursos.

A tabela a seguir mostra os fatores que facilitaram e dificultaram a adaptação dos enfermeiros na Unidade. 
Quadro1 - Opinião dos enfermeiros quanto ao fatores que facilitaram e dificultaram a sua adaptação dos enfermeiros na unidade. São Paulo, 1993

\begin{tabular}{|c|c|c|}
\hline Fatores & $\mathrm{N}^{\mathrm{O}}$ & de Enfermeiros \\
\hline $\begin{array}{l}\text { As informações, a receptividade e } \\
\text { o apoio do grupo de trabalho }\end{array}$ & & 08 \\
\hline $\begin{array}{l}\text { O esclarecimento prévio da dinâ - } \\
\text { mica do setor }\end{array}$ & & 01 \\
\hline $\begin{array}{l}\text { Muita informação em curto espaço de } \\
\text { tempo }\end{array}$ & & 02 \\
\hline Falta de conhecimento da planta física & & 02 \\
\hline Super lotação da Unidade & & 02 \\
\hline $\begin{array}{l}\text { Dificuldades pessoais tais como } \\
\text { inexperiência e insegurança }\end{array}$ & & 02 \\
\hline $\begin{array}{l}\text { O período de treinamento ser de ma- } \\
\text { nhã para quem irá trabalhar em ou - } \\
\text { tros turnos }\end{array}$ & & 01 \\
\hline Informatização & & 01 \\
\hline $\begin{array}{l}\text { Ficar escalada sozinha nos finais de } \\
\text { semana }\end{array}$ & & 01 \\
\hline TOTAL & & 20 \\
\hline
\end{tabular}

Observação: Houve mais de uma resposta por enfermeiro.

Pode ser dito que o acolhimento e o apoio da equipe, principalmente dos enfermeiros, e o fator mais importante para a integração do novo elemento na opinião dos enfermeiros recém-admitidos . Para DAYAL ${ }^{4}$ a adaptação do indivíduo ao trabalho, ou melhor, a sua socialização, é um processo dinâmico que depende do apoio recebido dos outros funcionários da empresa.

Pode se constatar ainda, pelo quadro acima, os diferentes fatores que prejudicam a orientação e a adaptação de novos elementos, e cujo conhecimento deverá auxiliar no planejamento de novos programas. Pelos dados obtidos eles estão ligados ao programa de integração, à organização e ao funcionamento das Unidades, assim como as dificuldades pessoais dos recém-admitidos. 


\section{CONSIDERAÇÕES FINAIS:}

Pelos resultados obtidos pode-se concluir que o programa de treinamento inicial tem cumprido seu papel, is to é, tem auxiliado os novos enfermeiros na adaptação ao trabalho. Porém, foi verificada a necessidade de algumas reformulações no conteúdo e no desenvolvimento do programa.

Ficou evidenciada a necessidade dos enfermeiros da Educação Continuada acompanharem também o desenvolvimento da fase do treinamento das Unidades, para avaliarem como o conteúdo, ministrado na Educação Continuada, deve ser reformulado para ter uma abordagem mais próxima a realidade das Unidades. Outro aspecto a ser considerado na reformulação é a criação de oportunidades para o retorno dos novos enfermeiros a Educação Continuada, para que estes, aposs vivenciarem a prática em seus locais de tabalho possam esclarecer suas dúvidas.

Deverão também, ser estudadas o aumento do período de orientação de alguns temas como a parte prática do SAE, a supervisão e liderança e a introdução ao sistema de informatização do hospital (HMS).

Em relação ao treinamento desenvolvido nas unidades deverá se estudar o aumento do tempo para a conclusão do cronograma de orientação específica pelos enfermeiros.

Deverão ser reforçados, junto às chefias e aos grupos de trabalho dos setores, os fatores facilitadores da adaptação do novo colega. Quanto aos fatores bloqueadores serão estudados, junto a equipe, as possíveis soluções.

Para finalizar, embora acreditemos na importância do programa de integração dos indivíduos nas organizações, sabemos que ele é um dos fatores que contribuem para isso. As condições de trabalho, tais como: salário, benefícios, jornada e clima organizacional entre outros, são fatores que também influenciam a permanência ou não do indivíduo na organização.

COAN, T.C.M. et al.. The newly admitted nurse's opinion about the integration training at the University Hospital. Rev.Esc.Enf.USP, v.30, n.2, p.187-203, aug, 1996.

The authors proposed themselves to get to know the newly admitted nurse's opinion, about the integration training done, in order to get subsidy to the evaluation and the improvement on this program.

UNITERMS: Nursing training. Nursing integration program. 


\section{REFERÊNCIAS BIBLIOGRÁFICAS}

01. AQUINO, E.P. de. Administração de recursos humanos: uma introdução. São Paulo, Atlas, 1980.

02. BERGSTEIN. N. A integraçāo do novo funcionário. In: BOOG, G.G. (coord.) Manual de treinamento e desenvolvimento. São Paulo, McGraw-Hill do Brasil, 1980. cap. 18, p. $273-80$.

03. CHIAvENATO, I. Recursos humanos na empresa. São Paulo, Atlas, 1989. v. 5.

04. DAYAL, I. Gerência de treinamento: textos, casos e exercícios de simulação. Rio de Janeiro, Livros Técnicos e Científicos. Såo Paulo, EDUSP, 1974.

05. FERNANDES. E.C. Sondagens de opinião interna como instrumento de informação. Rev. Adm, v. 27, n. 1, p. 36-48, 1992.

06. LEITE. M.M.J.; PEREIRA, L.L. Educação continuada em enfermagem. In: KURCGANT, P. (coord.). Administração em enfermagem. São Paulo, Ática, 1991. cap. 12, p.147-63.

07. SANTOS, O de B. Orientação e desenvolvimento do potencial humano. São Paulo, Pioneira, 1978.

08. SILVA, M.J.P. da; PEREIRA, L.L.; BENKO, M.A. Educação continuada: estratégia para o desenvolvimento de pessoal de enfermagem. Rio de Janeiro, Marques Saraiva, 1989.

09. WERTHER Jr., W.B.; DAVIS. K. Administração de pessoal e recursos humanos. São Paulo, McGraw-Hill do Brasil, 1983. 


\section{ANEXO 1}

\section{TREINAMENTO ADMISSIONAL - ENFERMEIRO}

\begin{tabular}{l}
\hline \multicolumn{1}{c}{ lo DIA } \\
início $8 \mathrm{~h} 30 \mathrm{~m}$ - Apresentação do grupo \\
início 9h30m - Hospital Universitário: \\
. Finalidade \\
. Filosofia \\
. População assistida \\
. Áreas de atendimento/ no de leitos /fluxo de pacientes \\
. Organograma \\
- Departamento de Enfermagem: \\
. Filosofia \\
. Comissão de Pesquisa Organizacional de Enfermagem(CPOE) \\
. Organograma
\end{tabular}

20 DIA

$02 \mathrm{~h} \quad-$ Orientações Trabalhistas

$01 \mathrm{~h} 30 \mathrm{~m} \quad$ - Visita HU

$01 \mathrm{~h} 30 \mathrm{~m}$ - Educação Continuada - Áreas de atuação

$01 \mathrm{~h}$ - Atribuições por função

$\begin{array}{ll}01 \mathrm{~h} 30 \mathrm{~m} & \text { - Etica Profissional } \\ 01 \mathrm{~h} 30 \mathrm{~m} & \text { - Relacionamento Interperssoal } \\ 03 \mathrm{~h} & \text { - Supervisão e Liderança }\end{array}$

4o DIA

04h - CCIH/Isolamento/Precauções Universais

01h - Padronização do uso de luvas

01h - Administração de Recursos Materiais do DE

\section{5o DIA}

06h - Cálculo de Drogas e Soluções (Resolução da apostila + correção)

\section{6o DIA}

04h - Introdução ao Sistema HMS

02h - Programa de Auditoria 


\section{7o DIA}

02h - Sistema de Assistência de Enfermagem (SAE)

04h - Historico de Enfermagem / Entrevista

- Lista de Problemas

- Evolução de Enfermagem

- Prescrição de Enfermagem

80 DIA

06h - Exame Físico - Leitura da Apostila + Discussão

9o DIA

03h - Prática do SAE na unidade de lotação

03h - Discussão da Prática

. Consulta de Enfermagem

. Anotação de Enfermagem 